\title{
Is COVID-19 a New Hematologic Disease?
}

\author{
Benjamin Debuc ${ }^{1} \cdot$ David M. Smadja ${ }^{2,3}$ \\ Published online: 12 May 2020 \\ (C) Springer Science+Business Media, LLC, part of Springer Nature 2020
}

\begin{abstract}
SARS-CoV-2 viruses are positive single-stranded RNA viruses, whose infection can be asymptomatic or lead to the coronavirus disease 2019 (Covid-19). Covid-19 is a respiratory infection with a significant impact on the hematopoietic system and hemostasis leading to several cardiovascular complications. Hematologic consequences of this new infection allowed medical community to start new treatment approaches concerning infection going from targeted anti-inflammatory drugs to anticoagulation or stem cell therapies. A better understanding of Covid-19 pathophysiology, in particular hematological disorders, will help to choose appropriate treatment strategies.
\end{abstract}

Keywords SARS-CoV-2 · Covid-19 • Coagulopathy · D-dimers · Lymphopenia · Mesenchymal stem cells · Cytokine · Inflammation $\cdot$ Thrombosis $\cdot$ Stem cells

\section{Introduction}

From the end of 2019, an epidemic pneumonia caused by a new coronavirus occurred in Wuhan (Hubei Province) spread rapidly throughout China, evolving into a global pandemic $[1,2]$. Originally called new coronavirus 2019 (2019-nCoV), this virus was then officially named severe acute respiratory syndrome coronavirus 2 (SARS-CoV-2) by WHO. On January 30, 2020, the WHO declared the SARS-CoV-2 epidemic a public health emergency of international concern. Coronaviruses are a large family of viruses that some of them are more known such as Middle East Respiratory Syndrome (MERS-CoV) and Severe Acute Respiratory Syndrome (SARS-CoV).

SARS-CoV-2 viruses are positive single-stranded RNA viruses, whose infection can be asymptomatic or lead to the coronavirus disease 2019 (Covid-19). Covid-19 has a very large pattern of respiratory manifestations but also other

David M. Smadja

david.smadja@aphp.fr

1 Université de Paris, Plastic Surgery Department, AP-HP, Georges Pompidou European Hospital, Paris F-75015, France

2 Université de Paris, Innovative Therapies in Haemostasis, INSERM UMR-S 1140, Paris F-75006, France

3 Hematology Department and Biosurgical Research Lab (Carpentier Foundation), AP-HP, Georges Pompidou European Hospital, 20 rue Leblanc, Paris F-75015, France non-specific symptoms including fever, headache, hemoptysis, nausea, vomiting, and in particular diarrhea also previously detected in other coronavirus infections [3, 4]. Respiratory manifestations have a large spectrum of infectious signs from fever, dry cough, and dyspnea to pneumonia, pulmonary edema and acute respiratory distress syndrome (ARDS) requiring hospitalization in intensive care unit (ICU). The clinical spectrum of COVID19 varies from asymptomatic or mild disease (more than $80 \%$ of all cases) to respiratory failure that requires mechanical ventilation and to multiple organ dysfunction syndromes or failure. Patients requiring hospitalization in an intensive care unit have a high mortality rate. In terms of pathogenesis, SARS-CoV-2 recognizes the angiotensin converting enzyme 2 receptor (ACE2) by its spike protein. ACE2 is widely expressed in human tissues, including alveloar type 2 (AT2), capillary epithelium, the heart, liver, kidney, and endothelial cells [5]. ACE2 is also expressed in hematopoietic stem and progenitor cells [6]. ACE2 stimulates mobilization of progenitor cells from bone marrow, migration to areas of vascular damage and revascularization of ischemic areas in pathological conditions like hypoxic exercise [6-8]. SARS-CoV-2 also probably invades host cells via CD147 (also known as Basigin or EMMPRIN) [9]. This CD147 has been also identified as a red blood cell receptor for parasite Plasmodium Falciparum [10] and a vascular receptor for Neisseria meningitidis [11]. CD147 has been described as a marker of undifferentiated embryonic stem cells [12] and is also expressed on mesenchymal stem cells [13]. In addition, a research team from Germany revealed that the cellular serine protease TMPRSS2 for SARS-CoV-2 spike protein priming is 
also essential for the host cell entry and spread [14]. Currently, there is no specific treatment for Covid-19 and understanding effects and mechanism of SARS-CoV-2 invasion and dissemination will help to find new targets.

\section{SARS-CoV-2 Infection Induces Hematological Disorders}

Covid-19 is a respiratory infection with a significant impact on the hematopoietic system and hemostasis. The current hallmark of SARS-CoV-2 pathogenesis is the cytokine storm. Indeed, plasma concentrations of interleukin-6, interleukin$1 \beta$, TNF- $\alpha$ but also granulocyte colony stimulating factor (G-CSF) or interferon $\gamma$-inducible protein (IP10) seem very high in Covid-19 patients and even higher in intensive care unit (ICU) patients than non-ICU patients [15]. This cytokine release syndrome made relevant treatment by blocking cytokines or their receptors like tocilizumab and sarilumab that are humanized monoclonal mAb blocking Il-6 receptors [16] (CORIMUNO-SARI: NCT04324073; CORIMUNO-TOCI: NCT04331808) or Anakinra (ANA) that is a recombinant human decoy IL-1RA and therefore blocks IL- $1 \alpha$ and IL-1 $\beta$ (CORIMUNO-ANA: NCT04341584). This cytokine release syndrome in Covid-19 patients is associated to a decreased count of lymphocyte [15]. Lymphopenia is present in more than $80 \%$ of Covid-19 patients at entrance [1] and could predict disease severity of Covid-19 [17]. This lymphopenia has been related to a significant decrease in $\mathrm{T}$ cells (in particular $\mathrm{CD} 8+\mathrm{T}$ cells) and this significant decrease of T lymphocyte subset is positively correlated with in-hospital death and severity of illness [18]. B lymphocytes have also been proposed to be involved in Covid-19, since patients with Agammaglobulinemia lacking B lymphocytes had a mild clinical course in contrast to Covid-19 patients with Common Variable Immune Deficiency [19]. Since Human B Cell Memory through $\mathrm{CD} 27^{\text {dull }}$ and $\mathrm{CD} 27^{\text {bright }}$ epitope is different along age, B lymphocytes could be at the origin of difference responses between children and adults in early phase of Covid-19. Indeed, B cells from children are able to better generate natural antibodies with novel pathogens when compared to B cells from adults [20].

Coagulopathy have been described in Covid-19 as soon as first cases were described. In this context of inflammation burst, link between coagulopathy and inflammation can be related to thrombo-inflammation mechanisms. This thrombo-inflammation has been described in arterial and/or venous thrombosis but also in cancer associated thrombosis with the complex interplay between blood coagulation and inflammation [21]. Covid-19 infection is associated with a coagulopathy characterized by an increase in procoagulant factors such as fibrinogen, together with a strong increase of D-dimers that have been associated with a higher mortality [3,
22]. D-dimer levels above $1000 \mathrm{ng} / \mathrm{mL}$ were an independent risk factor of in-hospital death [3]. Coagulopathy was also found in fatal cases of Covid-19 patients, including a significant higher proportion of patients with D-dimers above 500 $\mathrm{ng} / \mathrm{mL}$ and prolonged prothrombin time (PT) in non-survivors [22]. This result was also found in another Chinese population where D-dimers were still associated with in-hospital mortality [23]. D-dimers dynamics can also reflect the severity, increased levels were more pronounced among severe cases [3]. In terms of prothrombotic signal, Zhang et al. described, three cases of thrombosis associated with antiphospholipid antibodies represented by anticardiolipin (aCL) and anti- $\beta 2$ glycoprotein I (aß2GPI)2 [24]. In a French series from Mulhouse, $45 \%$ of Covid-19 patients had a detect lupus anticoagulant (LA) positive according to ISTH recommendations while aCL or aß2GPI were only positive in 3 patients associated to LA [25]. All these procoagulant profile in Covid-19 patients encouraged International society of thrombosis and haemostasis to propose preventive anticoagulation [26] in hospitalized Covid-19 patients since it could decrease mortality [27]. The question of curative anticoagulation is currently an open question that needs to answer in clinical trials. Moreover, in contrast to other respiratory viral pneumonia, an increased pulmonary embolism incidence has been observed in Covid-19 patients [28]. SARS-CoV-2 virus has been shown to infect blood vessels and induce vascular damage in vitro and in vivo [29-31]. This endothelial lesion can be at the origin of coagulopathy and hemostasis activation that lead to thrombotic disorders observed in Covid-19 patients.

\section{Are Mesenchymal Stem Cells the Key Treatment for Covid-19?}

Currently, no specific drugs or vaccines are available to treat Covid-19 patients. The therapeutic strategies are only supportive. Immunomodulatory drugs to block cytokine release syndrome are only focus on one pathway at a time. Mesenchymal stem cells (MSCs) have been described to have strong immunomodulatory properties. These cells have capacity to migrate to inflammatory sites, having anti-inflammatory and antifibrotic effects. Thus, MSCs could offer a new therapeutic approach. Moreover, their regenerative potential in particular, in vascular dysfunction of Covid-19, could be a good point. Indeed, MSCs have been described as proangiogenic cells in peripheral arterial disease [32]. In this context, MSCs act as paracrine cells since no endothelial differentiation is observed within adult bone marrow derived MSCs [32]. The interaction between endothelial cells and MSCs has been demonstrated in extracellular matrices models [33, 34] and also in vivo [35]. In these models, MSCs support vasculogenic processes because of their ability to transdifferentiate in perivascular cells when they interact with endothelial cells $[33,36,37]$ but also their 
ability to interact directly with endothelial endoglin pathway [38]. Thus, we can speculate that MSCs could modulate inflammation around endothelial cells during Covid-19 and also cure endothelial dysfunction to accelerate their recovery after Covid-19 induced vascular lesion. Allogenic MSCs have clinical grade culture and are already available in banks of cryofrozen cells. MSCs have been tested in several clinical trials with an excellent safety record in particular in immune mediated inflammatory diseases, such as graft versus host disease (GVHD) and autoimmune diseases [39]. Thus, several clinical studies are ongoing to test efficacy of MSCs in Covid19 patients [40]. Leng et al. investigated whether MSC transplantation improves the outcome of 7 enrolled patients with COVID 19 pneumonia in China [41]. They found improvement in pulmonary function and symptoms of their patients 2 days after MSCs transplantation. Clinically they noticed decrease of the fever, dyspnea and respiratory rate. Concerning the laboratory results, they noticed recovery of lymphopenia and decrease of CRP, aspartic aminotransferase and myoglobin levels. Moreover, after intravenous injection of MSCs, a significant decrease in circulating TNF- $\alpha$ concentration was observed. This study demonstrated safety but now efficiency need to be tested in large clinical trials. Bari et al. also proposed that MSCs secretome could be formulated as a drug and administered by intravenous injection or after inhalation [42]. Since MSCs-derived extracellular vesicles are well known vector of growth factors and different type of RNA [43], MSCs secretome could constitute a new and smart approach for COVID19 pneumonia treatment, in particular for the ones requiring hospitalization in intensive care unit [42]. Moreover, gene expression profiling of mesenchymal stem cells showed that these cells are ACE2 and TMPRSS2 negative, making them a good cell target not influenced by SARS-CoV-2 infection [41]. In Paris (France), one clinical trial is ongoing with MSCs isolated from Wharton's jelly of the umbilical cord (Stroma-Cov-2 project: NCT04333368) to treat covid-19 intubated-ventilated patients presenting with Acute Respiratory Distress Syndrome of less than $96 \mathrm{~h}$. Three intravenous infusions of umbilical cord Wharton's jelly-derived mesenchymal stromal cells will be realized. Thus, pleiotropic MSCs therapeutic effects could help to cure all hematological effects of SARS-CoV-2: inflammation and coagulopathy by a vascular effect. If MSCs are an efficient treatment of Covid19 , other allogenic stem cells could be helpful in future of virus-inducible cytokine release syndrome: these are very small embryonic like stem cells (VSELs). Indeed, VSELs have the ability to support endothelial cells angiogenic potential [44] and also ability to have their own endothelial differentiation $[45,46]$. Therefore, VSELs have been proposed as a promising cell for cardiac and vascular regeneration [46-48]. Moreover, VSELs are able to give rise to hematopoietic cells and could counteract lymphopenia and side effects of inflammation on hematopoietic cells. VSELs have been also described to help regeneration of lungs [49, 50], thus VSELs injection could help fighting Covid-19 induced lung fibrosis. As prerequisite to clinical trials, it will be essential to have an expansion of these cells. Indeed, ability of VSEL to proliferate in vitro is very limited $[45,51]$. Thus, more research on this topic is required to stimulate proliferative capacity of VSELs without affecting their potential for differentiation.

\section{Conclusions}

In conclusion, situation of the Covid-19 outbreak is having clinical and pathophysiological profiles currently under investigations. Understanding pathophysiology will help to choose more appropriated treatment. Vaccination, reducing virus replication or its cell entrance by interacting with ACE2 and/or CD147 and/or TMPRSS2 will be a key point for treatment design. Other ideal approaches will be to treat both inflammation and coagulopathy disorders. Stem and progenitor cells could be recruited by huge cytokine burst induced by SARS-CoV-2 infection and this phenomenon needs to be studied in the next future. Moreover mesenchymal stem cell transplantation could be an outstanding response to both cytokine release syndrome and vascular disorders. This SARS$\mathrm{CoV}-2$ seems to have more than one trick up his sleeve and probably we'll need to combine several pharmacological and stem therapy to succeed in critical form of Covid-19.

Funding Information David M. Smadja' research on Covid-19 is supported by a grant from the French research national agency (Agence Nationale pour la Recherche) ANR SARCODO.

\section{Compliance with Ethical Standards}

Disclosure Authors have nothing to disclose.

\section{References}

1. Guan, W. J., Ni, Z. Y., Hu, Y., Liang, W. H., Ou, C. Q., He, J. X., et al. (2020). Clinical characteristics of coronavirus disease 2019 in China. The New England Journal of Medicine.

2. Ratajczak, M. Z. (2020). How to "Detronize" virus in crown questions to current stem cell therapies. Stem Cell Reviews and Reports.

3. Zhou, F., Yu, T., Du, R., Fan, G., Liu, Y., Liu, Z., et al. (2020). Clinical course and risk factors for mortality of adult inpatients with COVID-19 in Wuhan, China: a retrospective cohort study. Lancet.

4. D'Amico, F., Baumgart, D. C., Danese, S., \& Peyrin-Biroulet, L. (2020). Diarrhea during COVID-19 infection: pathogenesis, epidemiology, prevention and management. Clinical Gastroenterology and Hepatology.

5. Hamming, I., Timens, W., Bulthuis, M. L., Lely, A. T., Navis, G., \& van Goor, H. (2004). Tissue distribution of ACE2 protein, the functional receptor for SARS coronavirus. A first step in understanding SARS pathogenesis. The Journal of Pathology, 203(2), 631-637. 
6. Jarajapu, Y. P. (2020). Targeting ACE2/Angiotensin-(1-7)/Mas receptor axis in the vascular progenitor cells for cardiovascular diseases. Molecular Pharmacology.

7. Joshi, S., Mahoney, S., Jahan, J., Pitts, L., Hackney, K. J., \& Jarajapu, Y. P.. (2020). Blood Flow Restriction Exercise Stimulates Mobilization of Hematopoietic Stem/Progenitor Cells and Increases the Circulating ACE2 Levels in Healthy adults. Journal of Applied Physiology (Bethesda, MD: 1985).

8. Heringer-Walther, S., Eckert, K., Schumacher, S. M., Uharek, L., Wulf-Goldenberg, A., Gembardt, F., et al. (2009). Angiotensin-(17) stimulates hematopoietic progenitor cells in vitro and in vivo. Haematologica, 94(6), 857-860.

9. Ulrich, H., \& Pillat, M. M. (2020). CD147 as a Target for COVID19 Treatment: Suggested Effects of Azithromycin and Stem Cell Engagement. Stem Cell Reviews and Reports.

10. Crosnier, C., Bustamante, L. Y., Bartholdson, S. J., Bei, A. K., Theron, M., Uchikawa, M., et al. (2011). Basigin is a receptor essential for erythrocyte invasion by Plasmodium falciparum. Nature, 480(7378), 534-7.

11. Bernard, S. C., Simpson, N., Join-Lambert, O., Federici, C., LaranChich, M. P., Maissa, N., et al. (2014). Pathogenic Neisseria meningitidis utilizes CD147 for vascular colonization. Nature Medicine, 20(7), 725-731.

12. Higashi, K., Yagi, M., Arakawa, T., Asano, K., Kobayashi, K., Tachibana, T., et al. (2015 Feb). A novel marker for undifferentiated human embryonic stem cells. Monoclonal Antibodies in Immunodiagnosis and Immunotherapy, 34(1), 7-11.

13. Amati, E., Perbellini, O., Rotta, G., Bernardi, M., Chieregato, K., Sella, S., et al. (2018). High-throughput immunophenotypic characterization of bone marrow- and cord blood-derived mesenchymal stromal cells reveals common and differentially expressed markers: identification of angiotensin-converting enzyme (CD143) as a marker differentially expressed between adult and perinatal tissue sources. Stem Cell Research \& Therapy, 9(1), 10.

14. Hoffmann, M., Kleine-Weber, H., Schroeder, S., Kruger, N., Herrler, T., Erichsen, S., et al. (2020). SARS-CoV-2 cell entry depends on ACE2 and TMPRSS2 and is blocked by a clinically proven protease inhibitor. Cell.

15. Lu, R., Zhao, X., Li, J., Niu, P., Yang, B., Wu, H., et al. (2020). Genomic characterisation and epidemiology of 2019 novel coronavirus: implications for virus origins and receptor binding. Lancet, 395(10224), 565-574

16. Michot, J. M., Albiges, L., Chaput, N., Saada, V., Pommeret, F., Griscelli, F., et al. (2020). Tocilizumab, an anti-IL6 receptor antibody, to treat Covid-19-related respiratory failure: a case report. Annals of Oncology.

17. Tan, L., Wang, Q., Zhang, D., Ding, J., Huang, Q., Tang, Y. Q., et al. (2020). Lymphopenia predicts disease severity of COVID-19: a descriptive and predictive study. Signal Transduction and Targeted Therapy, 5, 33.

18. Xu, B., Fan, C. Y., Wang, A. L., Zou, Y. L., Yu, Y. H., He, C., et al. (2020). Suppressed T cell-mediated immunity in patients with COVID-19: a clinical retrospective study in Wuhan, China. The Journal of Infection.

19. Quinti, I., Lougaris, V., Milito, C., Cinetto, F., Pecoraro, A., Mezzaroma, I., et al. (2020). A possible role for B cells in COVID-19?: lesson from patients with Agammaglobulinemia. The Journal of Allergy and Clinical Immunology.

20. Grimsholm, O., Piano Mortari, E., Davydov, A. N., Shugay, M., Obraztsova, A. S., Bocci, C., et al. (2020). The interplay between CD27(dull) and CD27(bright) B cells ensures the flexibility, stability, and resilience of human B cell memory. Cell Reports, 30(9), 2963-2977 e6.

21. d'Alessandro, E., Becker, C., Bergmeier, W., Bode, C., Bourne, J. H., Brown, H., et al. (2020). Thrombo-Inflammation in Cardiovascular Disease: An Expert Consensus Document from the Third Maastricht Consensus Conference on Thrombosis. Thrombosis and Haemostasis, 120(4), 538-564.

22. Tang, N., Li, D., Wang, X., \& Sun, Z. (2020). Abnormal Coagulation parameters are associated with poor prognosis in patients with novel coronavirus pneumonia. Journal of Thrombosis and Haemostasis.

23. Han, H., Yang, L., Liu, R., Liu, F., Wu, K. L., Li, J., et al. (2020). Prominent changes in blood coagulation of patients with SARSCoV-2 infection. Clinical Chemistry and Laboratory Medicine.

24. Zhang, Y., Xiao, M., Zhang, S., Xia, P., Cao, W., Jiang, W., et al. (2020). Coagulopathy and antiphospholipid antibodies in patients with Covid-19. The New England Journal of Medicine, 382(17), e38.

25. Harzallah, I., Debliquis, A., \& Drenou, B. (2020). Lupus anticoagulant is frequent in patients with Covid-19. Journal of Thrombosis and Haemostasis.

26. Thachil, J., Tang, N., Gando, S., Falanga, A., Cattaneo, M., Levi, M., et al. (2020). ISTH interim guidance on recognition and management of coagulopathy in COVID-19. Journal of Thrombosis and Haemostasis. In press.

27. Tang, N., Bai, H., Chen, X., Gong, J., Li, D., \& Sun, Z. (2020). Anticoagulant treatment is associated with decreased mortality in severe coronavirus disease 2019 patients with coagulopathy. Journal of Thrombosis and Haemostasis.

28. Poissy, J., Goutay, J., Caplan, M., Parmentier, E., Duburcq, T., Lassalle, F., et al. (2020). Pulmonary embolism in COVID-19 patients: awareness of an increased prevalence. Circulation.

29. Varga, Z., Flammer, A. J., Steiger, P., Haberecker, M., Andermatt, R., Zinkernagel, A. S., et al. (2020). Endothelial cell infection and endotheliitis in COVID-19. Lancet.

30. Monteil, V., Kwon, H., Prado, P., Hagelkruys, A., Wimmer, R. A., Stahl, M., et al. (2020). Inhibition of SARS-CoV-2 infections in engineered human tissues using clinical-grade soluble human ACE2. Cell.

31. Qanadli, S. D., Beigelman-Aubry, C., \& Rotzinger, D. C. (2020). Vascular changes detected with thoracic $\mathrm{CT}$ in coronavirus disease (COVID-19) might be significant determinants for accurate diagnosis and optimal patient management. AJR. American Journal of Roentgenology. W1.

32. Smadja, D. M., d'Audigier, C., Guerin, C. L., Mauge, L., Dizier, B., Silvestre, J. S., et al. (2012). Angiogenic potential of BM MSCs derived from patients with critical leg ischemia. Bone Marrow Transplantation, 47(7), 997-1000.

33. Melero-Martin, J. M., De Obaldia, M. E., Kang, S. Y., Khan, Z. A., Yuan, L., Oettgen, P., et al. (2008). Engineering robust and functional vascular networks in vivo with human adult and cord bloodderived progenitor cells. Circulation Research, 103(2), 194-202.

34. Smadja, D. M., Levy, M., Huang, L., Rossi, E., Blandinieres, A., Israel-Biet, D., et al. (2015). Treprostinil indirectly regulates endothelial colony forming cell angiogenic properties by increasing VEGF-A produced by mesenchymal stem cells. Thrombosis and Haemostasis, 114(4).

35. Rossi, E., Goyard, C., Cras, A., Dizier, B., Bacha, N., Lokajczyk, A., et al. (2017). Co-injection of mesenchymal stem cells with endothelial progenitor cells accelerates muscle recovery in hind limb ischemia through an endoglin-dependent mechanism. Thrombosis and Haemostasis, 117(10), 1908-1918.

36. Melero-Martin, J. M., Khan, Z. A., Picard, A., Wu, X., Paruchuri, S., \& Bischoff, J. (2007). In vivo vasculogenic potential of human blood-derived endothelial progenitor cells. Blood, 109(11), 47614768.

37. Au, P., Tam, J., Fukumura, D., \& Jain, R. K. (2008). Bone marrowderived mesenchymal stem cells facilitate engineering of longlasting functional vasculature. Blood, 111(9), 4551-4558.

38. Rossi, E., Bernabeu, C., \& Smadja, D. M. (2019). Endoglin as an adhesion molecule in mature and progenitor endothelial cells: a 
function beyond TGF-beta. Frontiers in Medicine (Lausanne), 6, 10.

39. Shammaa, R., El-Kadiry, A. E., Abusarah, J., \& Rafei, M. (2020). Mesenchymal Stem Cells Beyond Regenerative Medicine. Frontiers in Cell and Development Biology, 8, 72.

40. Golchin, A., Seyedjafari, E., \& Ardeshirylajimi, A. (2020). Mesenchymal stem cell therapy for COVID-19: present or future. Stem Cell Reviews and Reports.

41. Leng, Z., Zhu, R., Hou, W., Feng, Y., Yang, Y., Han, Q., et al. (2020). Transplantation of ACE2(-) Mesenchymal Stem Cells Improves the Outcome of Patients with COVID-19 Pneumonia. Aging and Disease, 11(2), 216-228.

42. Bari, E., Ferrarotti, I., Saracino, L., Perteghella, S., Torre, M. L., \& Corsico, A. G. (2020). Mesenchymal stromal cell secretome for severe COVID-19 infections: premises for the therapeutic use. Cells, 9(4).

43. Ratajczak, M. Z., Kucia, M., Jadczyk, T., Greco, N. J., Wojakowski, W., Tendera, M., et al. (2012). Pivotal role of paracrine effects in stem cell therapies in regenerative medicine: can we translate stem cell-secreted paracrine factors and microvesicles into better therapeutic strategies? Leukemia, 26(6), 1166-1173.

44. Guerin, C. L., Rossi, E., Saubamea, B., Cras, A., Mignon, V., Silvestre, J. S., et al. (2017). Human very small embryonic-like cells support vascular maturation and therapeutic revascularization induced by endothelial progenitor cells. Stem Cell Reviews, 13(4), $552-560$.

45. Guerin, C. L., Loyer, X., Vilar, J., Cras, A., Mirault, T., Gaussem, P., et al. (2015). Bone-marrow-derived very small embryonic-like stem cells in patients with critical leg ischaemia: evidence of vasculogenic potential. Thrombosis and Haemostasis, 113(5), 1084-1094.
46. Smadja, D. M. (2017). Bone marrow very small embryonic-like stem cells: new generation of autologous cell therapy soon ready for prime time? Stem Cell Reviews, 13(2), 198-201.

47. Dawn, B., Tiwari, S., Kucia, M. J., Zuba-Surma, E. K., Guo, Y., Sanganalmath, S. K., et al. (2008). Transplantation of bone marrowderived very small embryonic-like stem cells attenuates left ventricular dysfunction and remodeling after myocardial infarction. Stem Cells, 26(6), 1646-1655.

48. Wojakowski, W., Tendera, M., Kucia, M., Zuba-Surma, E., Milewski, K., Wallace-Bradley, D., et al. (2010). Cardiomyocyte differentiation of bone marrow-derived Oct-4 + CXCR4 + SSEA-1 + very small embryonic-like stem cells. International Journal of Oncology, 37(2), 237-247.

49. Guerin, C. L., Blandinieres, A., Planquette, B., Silvestre, J. S., Israel-Biet, D., Sanchez, O., et al. (2017). Very Small Embryoniclike Stem Cells Are Mobilized in Human Peripheral Blood during Hypoxemic COPD Exacerbations and Pulmonary Hypertension. Stem Cell Reviews, 13(4), 561-566.

50. Kassmer, S. H., Jin, H., Zhang, P. X., Bruscia, E. M., Heydari, K., Lee, J. H., et al. (2013). Very small embryonic-like stem cells from the murine bone marrow differentiate into epithelial cells of the lung. Stem Cells, 31(12), 2759-2766.

51. Ratajczak, M. Z., Ratajczak, J., Suszynska, M., Miller, D. M., Kucia, M., \& Shin, D. M. A (2017). Novel view of the adult stem cell compartment from the perspective of a quiescent population of very small embryonic-like stem cells. Circulation Research, 120(1), 166-178.

Publisher's Note Springer Nature remains neutral with regard to jurisdictional claims in published maps and institutional affiliations. 